\title{
Roles of aminoacyl-tRNA synthetase-interacting multi-functional proteins in physiology and cancer
}

\author{
Zheng Zhou', Bao Sun ${ }^{2,3}$, Shiqiong Huang ${ }^{4}$, Dongsheng Yu (ib) and Xiaochuan Zhang ${ }^{1}$
}

\begin{abstract}
Aminoacyl-tRNA synthetases (ARSs) are an important class of enzymes with an evolutionarily conserved mechanism for protein synthesis. In higher eukaryotic systems, eight ARSs and three ARS-interacting multi-functional proteins (AIMPs) form a multi-tRNA synthetase complex (MSC), which seems to contribute to cellular homeostasis. Of these, AIMPs are generally considered as non-enzyme factors, playing a scaffolding role during MSC assembly. Although the functions of AIMPs are not fully understood, increasing evidence indicates that these scaffold proteins usually exert tumor-suppressive activities. In addition, endothelial monocyte-activating polypeptide II (EMAP II), as a cleavage product of AIMP1, and AIMP2-DX2, as a splice variant of AIMP2 lacking exon 2, also have a pivotal role in regulating tumorigenesis. In this review, we summarize the biological functions of AIMP1, EMAP II, AIMP2, AIMP2-DX2, and AIMP3. Also, we systematically introduce their emerging roles in cancer, aiming to provide new ideas for the treatment of cancer.
\end{abstract}

\section{Facts}

AIMPs have various biological functions in addition to their roles as scaffolds in the MSC.

AIMPs and their variants are related to the occurrence and development of cancer.

Understanding of the molecular mechanisms linking AIMPs to cancer can contribute to identify new potential antitumoral strategies.

\section{Open questions}

Does the dysregulation of AIMPs in cancer affect the structure and function of the MSC?

What is the molecular mechanism by which AIMPs

Correspondence: Dongsheng Yu (dongshyu@163.com) or

Xiaochuan Zhang (xiaochuanzhang1990@163.com)

'Department of Chinese Medicine, The First Affiliated Hospital of Zhengzhou University, Zhengzhou 450000, China

${ }^{2}$ Department of Clinical Pharmacology, Xiangya Hospital, Central South University, Changsha 410000, China

Full list of author information is available at the end of the article

These authors contributed equally: Zheng Zhou, Bao Sun

Edited by: F. Pentimalli regulate tumorigenesis?

Is there potential for practical clinical applications based on findings concerning AIMPs in the context of cancer?

\section{Introduction}

Aminoacyl-tRNA synthetases (ARSs) are essential enzymes that participate in protein synthesis by catalyzing the activation of amino acids and linking them to their cognate transfer RNAs (tRNAs). In mammals, ARSs usually exist in free form or in the form of a multi-tRNA synthetase complex (MSC), and the latter consists of eight ARSs and three non-enzymatic ARS-interacting multifunctional proteins (AIMP1/p43, AIMP2/p38, and AIMP3/p18) ${ }^{1}$. Among them, AIMPs are generally considered as auxiliary proteins and play a role in scaffolding during MSC assembly ${ }^{2}$. In fact, AIMPs have several appended domains or motifs, which are involved in MSC formation and mediation of new functions ${ }^{1}$ (Fig. 1a). Meanwhile, AIMPs are closely linked with each other, and each of them has its preferable interacting ARSs in the $\mathrm{MSC}^{3-6}$. For example, X-ray crystallography confirmed that a ternary subcomplex consisting of aspartyl-tRNA synthetase (DRS), glutamyl-prolyl-tRNA synthetase 


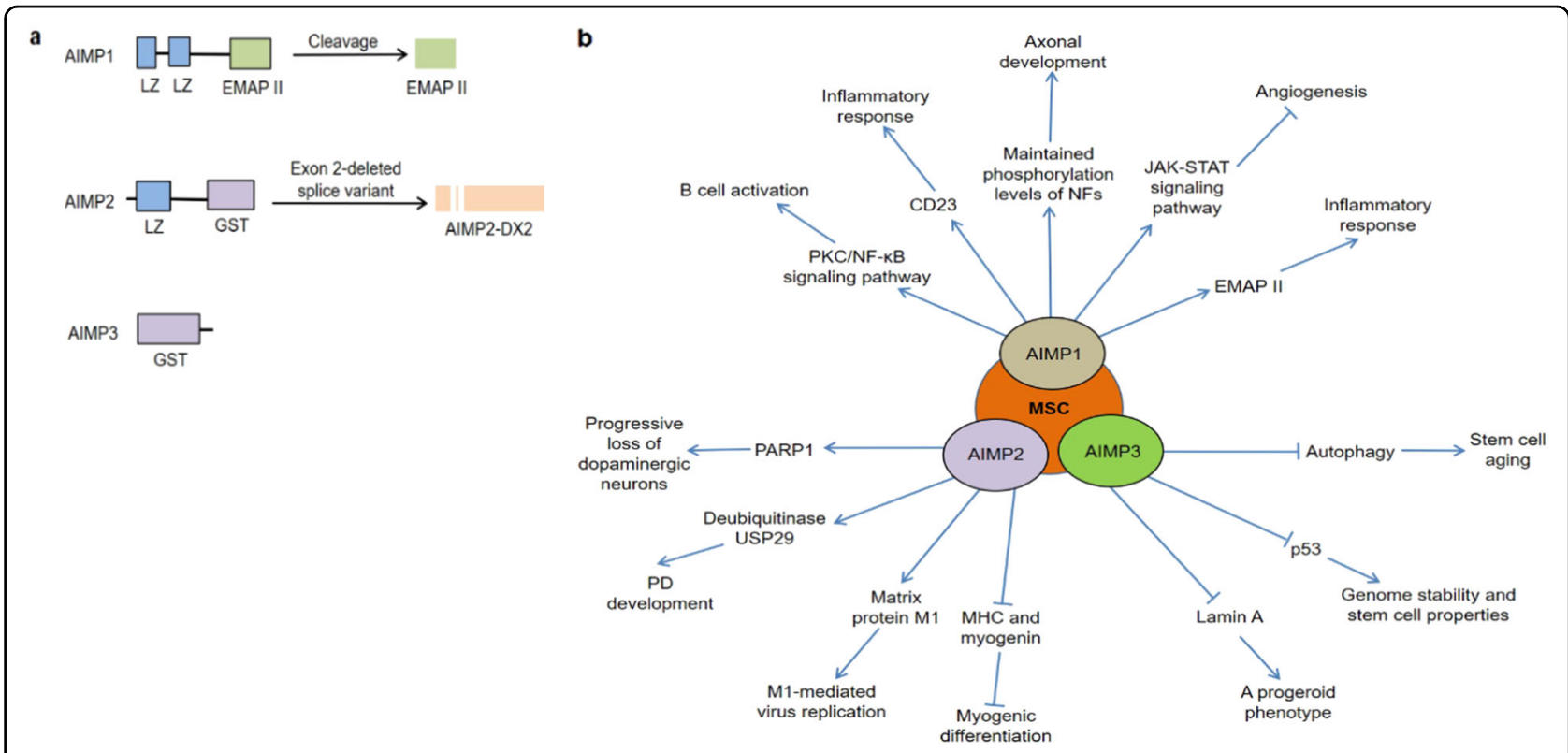

Fig. 1 Structures and biological functions of AIMPs. a Major domains and variants of AIMPs. $\mathbf{b}$ Various biological functions of AIMPs.

(EPRS) and AIMP2 provided a key architecture in the MSC $^{7}$. In this subcomplex, AIMP2 interacted with EPRS mainly through the heterodimerization of glutathione $S$ transferase (GST) domains, while DRS bound to AIMP2 by hydrogen bonds between the $\alpha 7-\beta 9$ loop of DRS and the $\beta 2-\alpha 2$ loop of AIMP2 $2_{\mathrm{GST}}$. Cho et al. found that four components, methionyl-tRNA synthetase (MRS), EPRS, AIMP2, and AIMP3, were assembled via a heterotetrameric complex structure of the GST domains in the human $\mathrm{MSC}^{8}$. Moreover, the full-length AIMP1 bound to AIMP3, and the N-terminus of AIMP1 bound to the GST-L domain of EPRS in the MSC ${ }^{9}$.

Interestingly, accumulating evidence has identified that AIMPs participate in various physiological and pathological processes as multifaceted molecules ${ }^{10-13}$. It was reported that the downregulation of AIMP1 enhanced transforming growth factor- $\beta$ (TGF- $\beta$ ) signal by inducing the phosphorylation of Smad family member 2/3 (Smad2/ 3 ), which in turn promoted the chondrogenic potential of dedifferentiated/degenerated chondrocytes ${ }^{14}$. Furthermore, patients with a homozygous c.105C >A (p.Tyr35Ter) mutation in AIMP2 showed microcephaly, seizures, mental retardation, and spastic quadriplegia ${ }^{15}$. Recent studies found that AIMP3 could maintain genomic integrity through the DNA repair process, and the adult mice with AIMP3 deletion developed an acute radiation syndrome-like phenotype ${ }^{16}$. Strikingly, AIMPs are also related to tumorigenesis ${ }^{17-19}$, which suggested that studying the additional functions of AIMPs in the context of cancer could expand our understanding of tumorigenesis. Here, we not only summarize the biological functions of AIMP1, EMAP II, AIMP2, AIMP2-DX2, and
AIMP3, but also focus on their emerging roles in regulating tumorigenesis, suggesting that their thorough study may provide new insights into cancer treatment.

\section{Biological functions of AIMPs}

Apart from their role as scaffolds in the MSC, AIMPs also have a variety of biological functions ${ }^{20-23}$. Notably, these non-canonical functions are closely related to immune regulation, nervous system functions, angiogenesis, viral replication, and genome stability (Fig. 1b).

\section{AIMPs and immune regulation}

AIMPs work as regulators or signaling molecules in some immune and inflammatory processes ${ }^{24-26}$. A previous study found that mammalian cells could specifically secrete the full-length AIMP1 without an apoptosis signal. In human monocytic THP-1 cells, AIMP1 could activate mitogen-activated protein kinase (MAPK) and nuclear factor $-\kappa B(N F-\kappa B)$ and induce the expression of multiple cytokines as well as chemokines, such as tumor necrosis factor (TNF), interleukin 8 (IL-8), monocyte chemotactic protein 1 (MCP-1) and macrophage inflammatory protein-1 $\alpha$ (MIP- $1 \alpha)$, which were generally regarded as the main factors inducing atherosclerosis ${ }^{27}$. Importantly, AIMP1 was highly expressed in the foam cells of atherosclerotic lesions, indicating that it might promote the development of atherosclerosis by inducing cytokines and chemokines. CD23 (FceRII) was thought to be a functional receptor molecule for AIMP1, which mediated the inflammatory response induced by AIMP1 $1^{28}$. During this process, AIMP1 bound to CD23 and subsequently induced TNF- $\alpha$ secretion by activating extracellular 
signal-regulated kinase 1/2 (ERK1/2) in THP-1 cells and peripheral blood mononuclear cells (PBMCs). Notably, a neutralizing antibody MHM6 inhibited the secretion of TNF- $\alpha$ induced by AIMP1 via competing with IgE for binding to CD23, suggesting that the interaction between AIMP1 and CD23 in monocytes might play an important role in autoimmune diseases. Recently, Kim et al. demonstrated that AIMP1 inhibited $\mathrm{T}$ cell receptor (TCR)-mediated CD4 $\mathrm{T}$ cell activation and proliferation ${ }^{29}$. AIMP1 weakened the TCR signal by interfering with lipid raft aggregation and thus reduced the phosphorylation of phospholipase $\mathrm{C} \gamma$ (PLCY) and phosphatidylinositol 3hydroxy kinase (PI3K) in CD4 T cells. At the same time, AIMP1 specifically enhanced the differentiation of regulatory $\mathrm{T}$ (Treg) cells, but had no effect on the differentiation of T helper type 1 (Th1), Th2, and Th17 cells.

EMAP II was first discovered to be secreted by tumor cells and could induce acute inflammatory response in vivo ${ }^{30}$. Subsequent research showed that AIMP1 was the precursor of EMAP II, which was released after AIMP1 cleavage ${ }^{31,32}$. Lee et al. revealed that EMAP II promoted the development of bronchopulmonary dysplasia (BPD) in mice by recruiting macrophages to aggravate the inflammatory state ${ }^{33}$. By further confirming the transcription characteristics of macrophages exposed to EMAP II, researchers found that the unique transcription profile in response to EMAP II was mainly mediated by janus kinase (JAK)-signal transducer and activator of transcription 3 (STAT3), providing a direction for the study of EMAP II-mediated inflammation ${ }^{34}$. Furthermore, TCR affinity controlled the differentiation process by regulating the AIMP3, IL-2R $\alpha$-chain (CD25) and guanylate binding protein $2(\mathrm{Gbp} 2)$ in naive $\mathrm{T}$ cells ${ }^{35}$. Specifically, low TCR affinity induced the expression of Gbp2, thereby promoting $\mathrm{T}$ follicular helper (Tfh) cell differentiation, while high TCR affinity induced the expression of AIMP3 and CD25, thereby promoting nonTfh cell formation.

\section{AIMPs and nervous system functions}

Pathogenic mutations in AIMP1 have been reported to be associated with neurological diseases, such as neurodegenerative disease, pontocerebellar hypoplasia, and intellectual disability ${ }^{36-38}$. For example, a homozygous c.917A>G (p.Asp306Gly) mutation in AIMP1 caused severe neurodegenerative phenotypes, including developmental delays, epilepsy and progressive microcephaly ${ }^{39}$. Meanwhile, magnetic resonance imaging (MRI) showed that the neuroimaging features were callosal atrophy and T2 hyperintensity in the superficial white matter, as well as preserved myelination in the periventricular and deep white matter structures. Of note, AIMP1-deficient mice showed axon degeneration in motor neurons, defects of neuromuscular junctions, motor dysfunction and muscular atrophy ${ }^{40}$. AIMP1 was mainly expressed in central neurons and specifically interacted with the rod domain of neurofilament-light subunit (NF-L). Moreover, AIMP1 was a negative regulator of NF phosphorylation, and its overexpression or depletion could change the phosphorylation level of NFs, leading to the NF network disassembly. Recently, $\mathrm{Xu}$ et al. discovered that the $\mathrm{N}$ terminus of AIMP1 was responsible for the binding to its $\mathrm{C}$ terminus and arginyl-tRNA synthetase (RARS), and it also colocalized to the NF-L subunit protein ${ }^{41}$. These findings suggest that AIMP1 plays an important role in NF assembly and axon maintenance, which provides a new idea for exploring the pathogenesis of neurological diseases.

Interestingly, AIMP2 was a Parkin substrate ${ }^{42}$. In the dopaminergic neuroblastoma-derived SH-SY5Y cell line, Parkin promoted the ubiquitylation and degradation of AIMP2. Importantly, the overexpression of Parkin significantly protected SH-SY5Y cells from AIMP2-induced cell death. Lee et al. showed that AIMP2 accumulation overactivated poly(ADP-ribose) polymerase-1 (PARP1), resulting in the PAR accumulation and progressive loss of dopaminergic neurons, suggesting that AIMP2-induced parthanatos contributed to dopaminergic cell death, and that PARP1 inhibitors might be used to delay the progression of Parkinson's disease (PD ${ }^{43}$. Conspicuously, vacuolar protein sorting-associated protein 35 (VPS35) prevented AIMP2-mediated PARP1 activation and cell death by facilitating lysosomal degradation of AIMP2, suggesting that VPS35 might be a potential target for relieving $\mathrm{PD}^{44}$. AIMP2 transcriptionally upregulated deubiquitinase ubiquitin-specific peptidase 29 (USP29), resulting in the accumulation of $160 \mathrm{kDa}$ myb-binding protein (MYBBP1A) in PD $^{45}$. The expression of USP29 and MYBBP1A was upregulated in the Parkin knockout SH-SY5Y cells and the ventral midbrain of AIMP2 transgenic mice, suggesting that MYBBP1A might contribute to the development of PD.

\section{Other biological functions}

A previous study by Park et al. demonstrated that AIMP1 played a dose-dependent biphasic role in angiogenesis $^{46}$. Low concentrations of AIMP1 activated matrix metalloproteinase 9 (MMP9) through ERK, leading to the migration of endothelial cells. Conversely, high concentrations of AIMP1 activated c-Jun N-terminal kinase (JNK), thereby inducing the apoptosis of endothelial cells. Mechanistically, the JAK-STAT signaling pathway mediated the inhibitory effect of AIMP1 on angiogenesis ${ }^{47}$. Furthermore, many studies have shown that EMAP II is an effective antiangiogenic cytokine ${ }^{48,49}$. In myocardial infarction mice treated with EMAP II antibody, researchers found upregulated angiogenesis-related biomarkers and increased endothelial cell, suggesting that 
EMAP II blockade improved cardiac function by inducing angiogenesis ${ }^{50}$.

In influenza A virus (IAV)-infected cells, the virus NS2 protein protected host AIMP2 from ubiquitin-mediated degradation $^{51}$. Subsequently, the accumulated AIMP2 promoted the conversion from ubiquitination to SUMOylation of matrix protein M1, thereby promoting M1-mediated virus replication. This finding suggests that AIMP2 plays an important role in IAV infection and may serve as a potential target for the treatment of influenza. Furthermore, researchers also discovered that AIMPs were associated with DNA damage. Liu et al. found that the rs12199241 of AIMP3 was significantly related to the levels of DNA damage in Chinese population ${ }^{52}$. Analogously, AIMP3 depletion in mouse embryonic stem cells blocked double-strand break repair, which resulted in the accumulation of DNA damage and genome instability ${ }^{53}$. In addition, AIMPs were also involved in other biological processes, such as glucose homeostasis, liver fibrosis, myogenic differentiation, and aging ${ }^{54-58}$.

\section{Roles of AIMPs in cancer}

Cancer is a serious threat to human health and life and is rapidly becoming a major global health burden. A growing number of reports have described the association between AIMPs and cancer, suggesting the potential significance of AIMPs in cancer biology.

\section{AIMP1 and cancer}

Lee et al. observed that AIMP1 had significant antitumor activity in a xenograft mouse model $^{59}$. The expression of AIMP1, AIMP2, and AIMP3 was downregulated in gastric and colorectal cancer, which might result in their inactivation of tumor suppressor functions and tumor development ${ }^{60}$. Moreover, RARS could regulate the secretion of AIMP1 in HeLa and MCF7 cell lines $^{61}$. In laryngeal squamous cell carcinoma (LSCC) tissues, AIMP1 and leukotriene A4 hydrolase (LTA4H) were upregulated and promoted the proliferation, migration, and invasion of LSCC cells ${ }^{62}$. Importantly, AIMP1 and LTA4H bound to fascin actin-bundling protein 1 (FSCN1), suggesting that their interaction might promote the progress of LSCC.

Particularly, AIMP1 plays an important regulatory role in tumor immunity. Based on the abundant immunophenotype in glioblastoma (GBM), Cheng et al. identified eight immune-related genes with prognostic value in $\mathrm{GBM}^{63}$. Of these, AIMP1, forkhead box O3 (FOXO3) and zinc finger and BTB domain containing 16 (ZBTB16) were defined as protective with $\mathrm{HR}<1$, whereas IL-6, IL10, chemokine ligand 18 (CCL18), Fc fragment of IgG receptor IIb (FCGR2B) and MMP9 were defined as risky with $H R>1$. Meaningfully, the researchers developed a local immune-related risk signature from these genes to distinguish cases as high or low risk of unfavorable prognosis.

Moreover, the absence of AIMP1 in bone marrowderived dendritic cells (BMDCs) reduced downstream Th1 polarization of $\mathrm{T}$ cells by impairing $\mathrm{p} 38$ MAPK signaling, which significantly weakened BMDC vaccinemediated protection against melanoma ${ }^{64}$. Meanwhile, The Cancer Genome Atlas (TCGA) database analysis showed that the expression of AIMP1 in nearly 9000 primary tumor samples was highly correlated with longterm survival. These results indicated that AIMP1 was critical for effective antitumor immunity. Kim et al. supported that AIMP1 activated natural killer (NK) cells through macrophages, which significantly inhibited lung metastasis of melanoma cells in vivo ${ }^{65}$. In this process, the direct contact between macrophages and NK cells was necessary for AIMP1-induced NK cell activation. AIMP1 also significantly promoted the secretion of TNF- $\alpha$ by macrophages, which partially supported the activation of NK cells. Interestingly, AIMP1 reduced the population of myeloid-derived suppressor cells (MDSCs) in the spleens and primary tumor sites of breast tumor-bearing mice and inhibited the expansion of MDSCs in tumor-conditioned media ${ }^{66}$. In addition, AIMP1 not only negatively regulated the inhibitory activity of MDSCs by reducing the production of IL-6, nitric oxide (NO), and arginase-1 (Arg-1), but also effectively attenuated the ability of MDSCs to suppress $\mathrm{T}$ cell proliferation and induce Treg cell differentiation in vivo. Further research discovered that the negative regulation of MDSC functions by AIMP1 might be related to the weakened activation of STATs, protein kinase B (Akt) and ERK. Collectively, these findings indicate that AIMP1 actively participates in tumor immunity through regulating various physiological processes, such as Th1 polarization, NK cell activity, and MDSC functions (Fig. 2).

\section{EMAP II and cancer}

Previous research found that EMAP II was a tumorsuppressive cytokine with antiangiogenic effects, which inhibited the primary and metastatic tumor growth and facilitated apoptosis in growing capillary endothelial cells $^{67}$. Low-dose EMAP II inhibited tumor growth by inducing defective autophagy and G2/M arrest in glioblastoma stem cells (GSCs) ${ }^{68}$. Mechanistically, EMAP II reduced the expression of phosphorylated PI3K and Akt with concomitant induction of FoxO1 activation. The specific knockout of FoxO1 greatly reduced the induction of autophagy and G2/M arrest, suggesting that the PI3K/ Akt/FoxO1 axis was involved in the anticancer effect of EMAP II in GSCs. Li et al. demonstrated that EMAP II inhibited the viability, migration, and tube formation of GBM-induced endothelial cells (GECs) by inducing autophagy, thereby inhibiting GBM-induced 


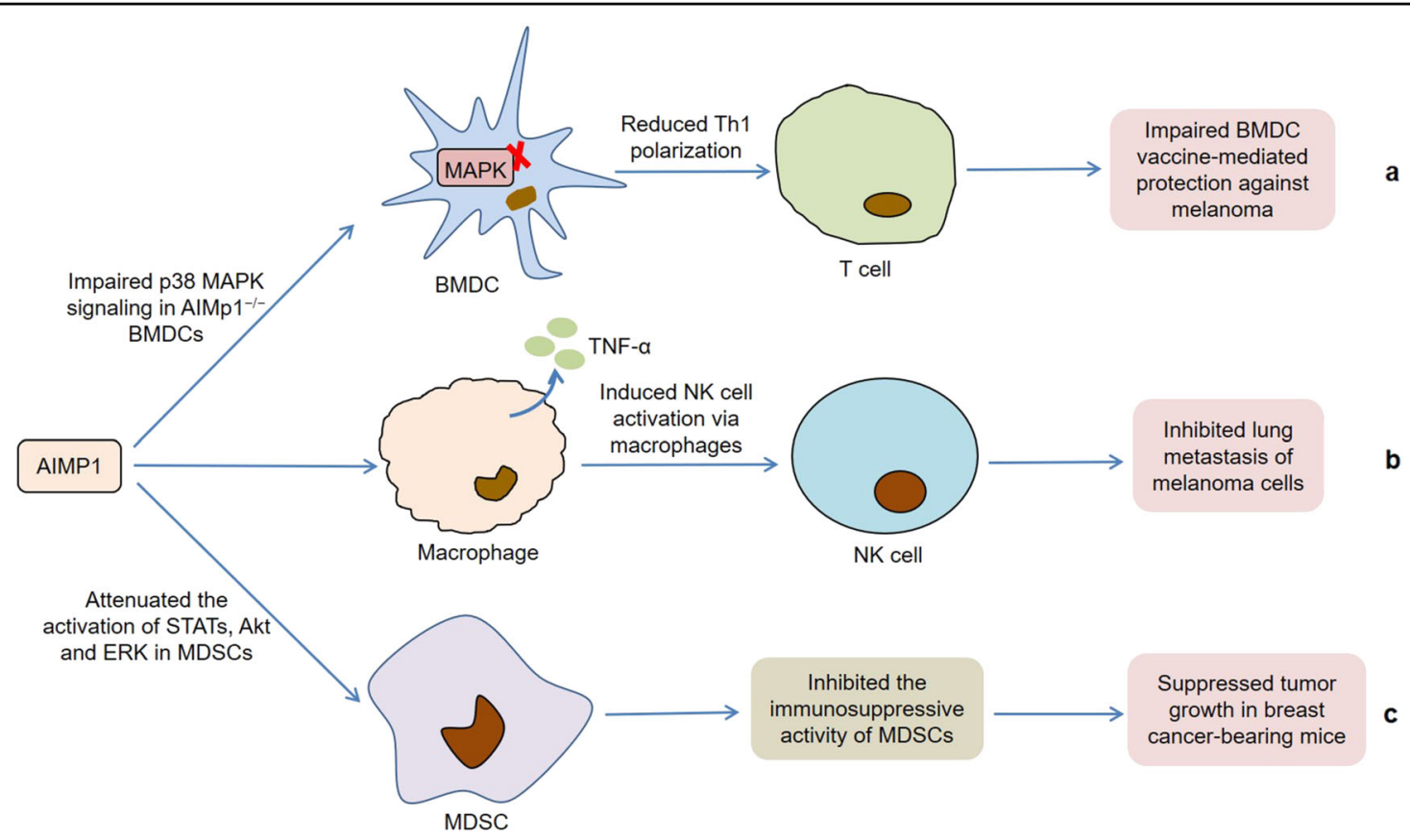

Fig. 2 Roles of AIMP1 in tumor immunity. a The absence of AIMP1 in BMDCs reduces Th1 polarization of T cells by impairing p38 MAPK signaling, which significantly impairs BMDC vaccine-mediated protection against melanoma. $\mathbf{b}$ AIMP1 activates NK cells through the direct contact between macrophages and NK cells, which inhibits lung metastasis of melanoma cells. Meanwhile, AIMP1 also promotes the secretion of TNF-a by macrophages, which partially supports the activation of NK cells. c AIMP1 inhibits the immunosuppressive activity of MDSCs by attenuating the activation of STATs, Akt, and ERK, thereby suppressing the tumor growth in breast cancer-bearing mice.

angiogenesis $^{69}$. GECs treated with EMAP II showed upregulated expression of microtubule-associated protein-1 light chain-3 (LC3) and sequestosome 1 (p62/ SQSTM1) and blockage of PI3K/Akt/mammalian target of rapamycin (mTOR) pathway. At the mechanistic level, EMAP II downregulated the expression of miR-96, which upregulated the expression of LC3 and p62/SQSTM1 by directly targeting unfolded protein response (UPR)-related proteins, such as glucose-regulated protein 78 (GRP78), eukaryotic translation initiation factor 2 alpha (eIF2 $\alpha$ ) and C/EBP homologous protein (CHOP). Analogously, low-dose EMAP II induced autophagy by downregulating the expression of miR-20a in human U-87 and $\mathrm{U}-251$ glioma cells ${ }^{70}$. In this process, miR-20a negatively regulated the expression of autophagy-related 5 (ATG5) and ATG7 by directly targeting their $3^{\prime}$-UTR, thereby activating the autophagy pathway.

In addition, EMAP II sensitized human melanoma to systemic TNF- $\alpha$ in vivo ${ }^{71}$. Mechanistic investigations found that the expression of TNF-R1 protein was not increased in human endothelial cells treated with EMAP II, but instead was redistributed from Golgi storage pools to cell membranes ${ }^{72}$. Meanwhile, EMAP II induced the membrane expression and mobilization of TNF-R1associated death domain (TRADD) protein. Intriguingly, EMAP II was associated with the permeability of blood- tumor barrier $(\mathrm{BTB})^{73-75}$. Liu et al. observed that EMAP II upregulated the expression of protein kinase C- $\alpha$ (PKC$\alpha)$ and increased its activity by inhibiting the expression of miR-330-3p, resulting in a decreased expression of tight junction (TJ)-related proteins including zonulae occludens-1 (ZO-1), occludin and claudin-5, as well as an increased permeability of $\mathrm{BTB}^{76}$. MiR-429 also mediated the effects of EMAP II on the permeability of $\mathrm{BTB}^{77}$. Specifically, EMAP II significantly upregulated the expression of miR-429, which not only inhibited the expression of ZO-1 and occludin by directly interacting with them, but also reduced the expression of TJ-related proteins by downregulating the expression and phosphorylation of p70 ribosomal protein S6 kinase (p70S6K). Furthermore, caveolae-mediated transcellular pathway was involved in the increased permeability of BTB induced by EMAP II in C6 glioma rats $^{78}$.

Interestingly, in addition to the various antitumor effects described above, EMAP II is also associated with immune escape by tumor cells. Recombinant EMAP II suppressed DNA synthesis and cell division in PBMCs and induced apoptosis in PBMCs and Jurkat cells ${ }^{79}$. Consistently, native EMAP II expressed on the surface of HT29 cells could activate caspase 8 in Jurkat cells, which led to their apoptosis. Youssef et al. pointed out that colorectal cancer cells secreted EMAP II under hypoxic 


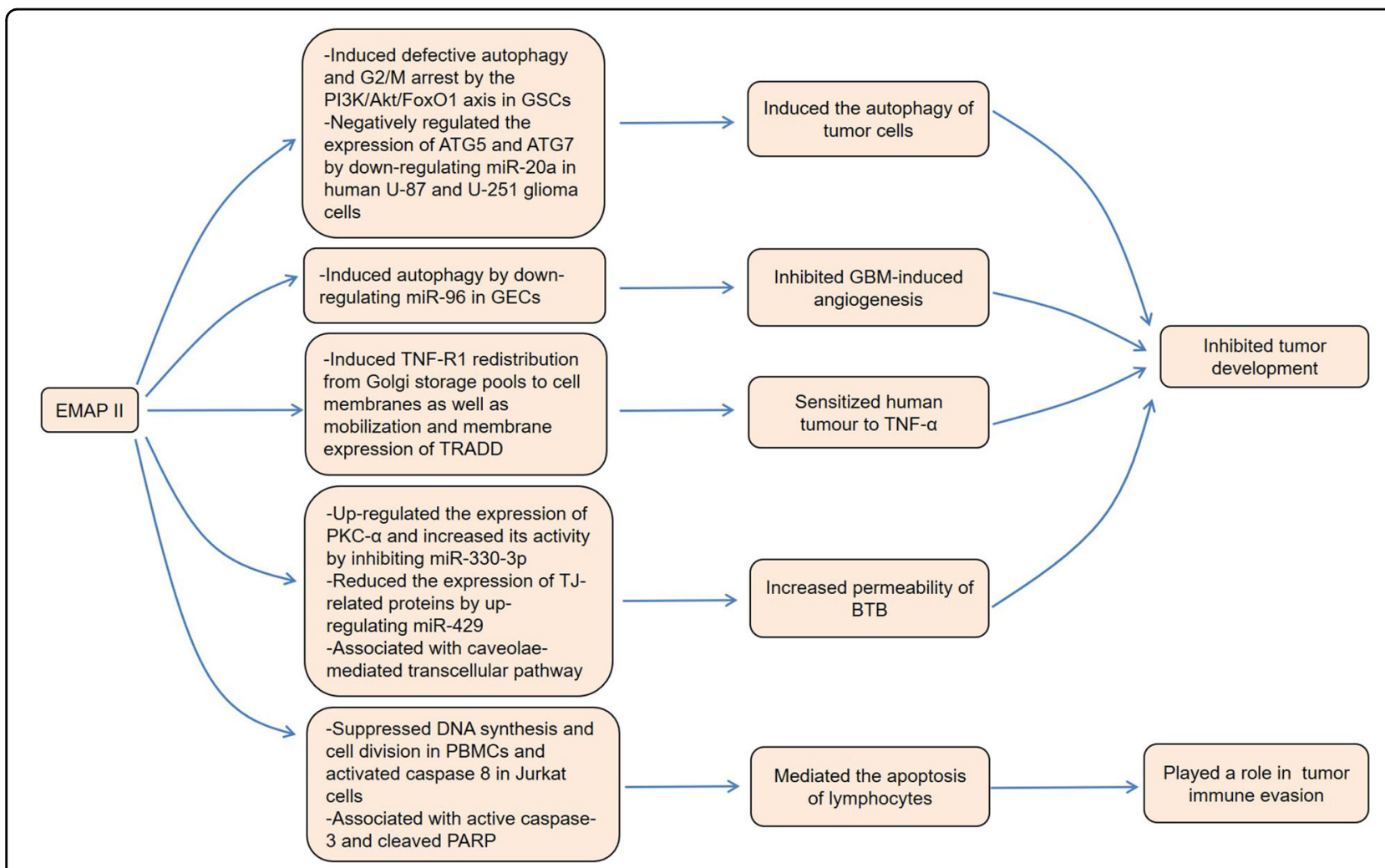

Fig. 3 .Roles of EMAP II in the occurrence and development of cancer. On the one hand, EMAP II exerts antitumor effects by inducing tumorcell autophagy, inhibiting angiogenesis, sensitizing tumor cells to TNF-a, and increasing the permeability of BTB. On the other hand, EMAP II promotestumor development by inducing lymphocyte apoptosis.

conditions, which mediated the apoptosis of tumorinfiltrating lymphocytes induced by hypoxia ${ }^{80}$. In conclusion, EMAP II not only exerts antitumor effects by inducing autophagy of tumor cells, inhibiting angiogenesis, sensitizing tumor cells to TNF- $\alpha$, and increasing the permeability of BTB, but also promotes the development of cancer by inducing lymphocyte apoptosis (Fig. 3).

\section{AIMP2 and cancer}

It is reported that AIMP2 usually shows tumorsuppressive activities (Fig. 4). A previous study by Choi et al. supported that the decreased AIMP2 levels in heterozygous AIMP2 mice provided greater sensitivity to multiple tumor formations, suggesting that AIMP2 could serve as a haploinsufficient tumor suppressor ${ }^{81}$. AIMP2deficient cells were resistant to DNA damage-induced cell death, while the overexpression of AIMP2 enhanced the sensitivity to apoptosis ${ }^{82}$. Upon DNA damage, AIMP2 was phosphorylated by JNK and dissociated from the MSC. Subsequently, the dissociated AIMP2 translocated to the nucleus and directly interacted with tumor suppressor $\mathrm{p} 53$, thereby inhibiting murine double minute 2 (MDM2)-mediated ubiquitination and degradation of p53. These findings indicate that AIMP2 can regulate cell death through p53. It is well known that TNF- $\alpha$ is closely related to tumorigenesis ${ }^{83,84}$. TNF- $\alpha$ induced cell death was decreased in AIMP2-deficient cells ${ }^{85}$. Conversely, exogenous supplementation of AIMP2 enhanced the apoptotic sensitivity to TNF- $\alpha$. Further studies shown that AIMP2 promoted the ubiquitin-dependent degradation of TNF receptor-associated factor 2 (TRAF2) by enhancing the recruitment of the E3 ligase c-IAP1 to TRAF2, thus mediating the pro-apoptotic activity of TNF- $\alpha$. This finding suggests that AIMP2 may be involved in tumor development by regulating the TNF- $\alpha$ signaling pathway.

Hemizygous deletion of AIMP2 increased the formation of adenoma in $\mathrm{Apc}^{\mathrm{Min} /+}$ mice and resulted in the proliferation of intestinal epithelial cells (IECs) in crypts and expansion of intestinal stem cell (ISC) compartments ${ }^{86}$. Further research found that AIMP2 disrupted the interaction between axis inhibition protein (AXIN) and Dishevelled-1 (DVL1) by binding to DVL1, which inhibited Wnt $/ \beta$-catenin signaling and therefore controlled ISC compartments and tumorigenesis. Moreover, TGF- $\beta$ induced AIMP2 expression and promoted its translocation to the nucleus, thereby participating in lung cell 


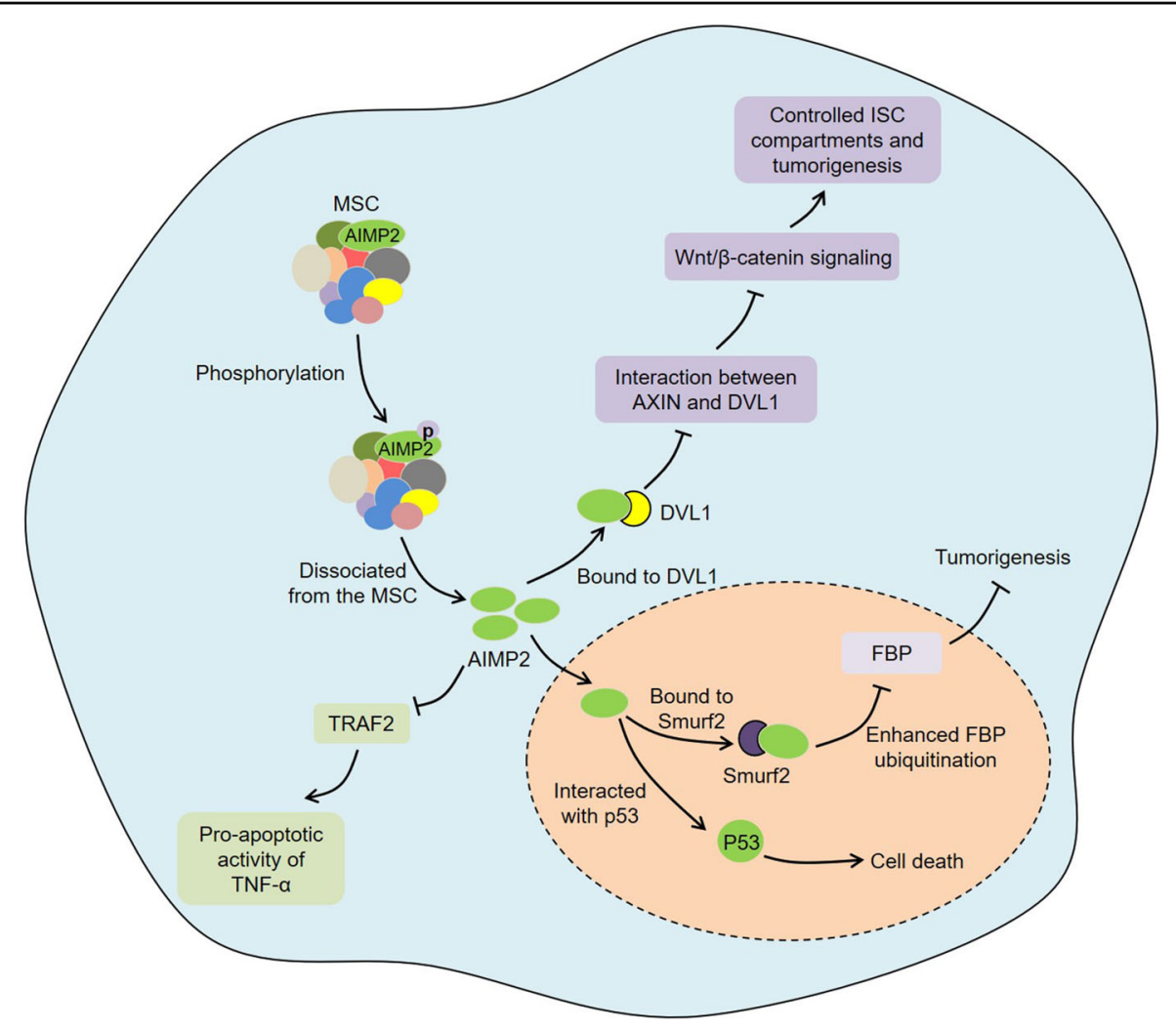

Fig. 4 Roles of AIMP2 in cancer. Upon DNA damage, AIMP2 is phosphorylated and dissociates from the MSC. Subsequently, the dissociated AIMP2 translocates to the nucleus and directly interacts with tumor suppressor p53. AIMP2 promotes the pro-apoptotic activity of TNF-a via ubiquitinmediated degradation of TRAF2. AIMP2 disrupts the interaction between AXIN and DVL1 by binding to DVL1, which inhibits Wnt/ $\beta$-catenin signaling and therefore controls ISC compartments and tumorigenesis. Furthermore, the dissociated AIMP2 binds to Smurf2, thereby enhancing the ubiquitination of FBP and inhibiting tumorigenesis.

differentiation ${ }^{87}$. Mechanistically, the AIMP2 in the nucleus interacted with FUSE-binding protein (FBP), which stimulated the ubiquitination and degradation of FBP, leading to the downregulation of c-Myc. Another study discovered that upon TGF- $\beta$ stimulation, AIMP2 was phosphorylated at S156 site by p38 MAPK and then dissociated from the MSC in HeLa cells ${ }^{88}$. The dissociated AIMP2 translocated to the nucleus and bound to Smad ubiquitin regulatory factor 2 (Smurf2), thereby enhancing the ubiquitination of FBP and inhibiting tumor formation. Notably, the S156A mutant in AIMP2 that inhibited its nuclear interaction with Smurf2 promoted tumorigenesis in vivo. Zhong et al. demonstrated that the RARS-mitotic arrest deficient-like 1 (MAD1L1) fusion protein interacted with AIMP2 to increase the expression of FBP, thereby promoting the occupation of the c-Myc promoter by FBP and subsequently inducing cancer stem cell (CSC)-like properties ${ }^{89}$. These data suggest that the development of drugs targeting the $\mathrm{FBP} / \mathrm{c}-\mathrm{Myc}$ axis via combinatorial therapy may be beneficial for certain types of cancer patients.

\section{AIMP2-DX2 and cancer}

AIMP2-DX2, as a splice variant of AIMP2 lacking exon 2 , was highly expressed in human lung cancer cells, and the ratio of AIMP2-DX2 to normal AIMP2 was increased with cancer progression ${ }^{90}$. Endogenous AIMP2-DX2 impaired the pro-apoptotic activity of AIMP2 through the competitive binding to p53 in lung cancer A549 cells. Interestingly, transgenic mice expressing AIMP2-DX2 showed increased sensitivity to lung tumorigenesis compared to the wild type counterpart. Jung et al. discovered that lung cancer patients with high AIMP2-DX2/AIMP2 autoantibody ratio had significantly shorter overall survival than those with low ratio, suggesting that AIMP2DX2 levels were related to the clinical outcome of lung cancer $^{91}$. Moreover, AIMP2-DX2 was highly expressed in chemoresistant ovarian cancer ${ }^{92}$. AIMP2-DX2 reduced the pro-apoptotic activity of TNF- $\alpha$ by competitively inhibiting the binding of AIMP2 to TRAF2, thereby contributing to the chemoresistance of ovarian cancer. Heat shock protein 70 (HSP70) was positively correlated with AIMP2-DX2 in lung cancer patient tissues ${ }^{93}$. 


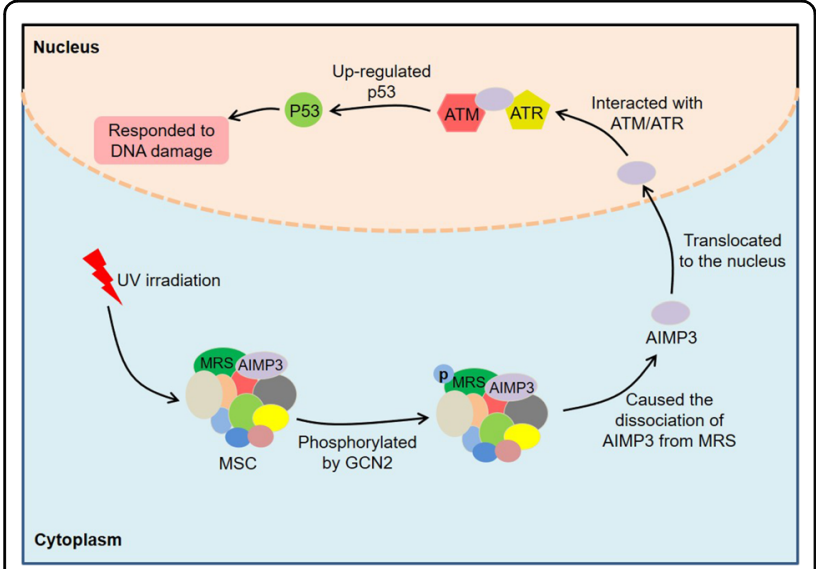

Fig. 5 Roles of AIMP3 in cancer. Under UV irradiation, MRS is phosphorylated at Ser662 by GCN2, causing a conformational change in MRS and the subsequent dissociation of AIMP3 from MRS. The dissociated AIMP3 translocates to the nucleus and upregulates p53 by directly interacting with the FAT domains of ATM/ATR, thereby responding to DNA damage.

Importantly, HSP70 could block the Seven in absentia homolog 1 (Siah1) binding and ubiquitination of AIMP2DX2 by specifically recognizing and stabilizing AIMP2DX2, leading to an increase in AIMP2-DX2 levels. X-ray crystallography and NMR analysis revealed that HSP70 bound to the amino $(\mathrm{N})$-terminal flexible region and glutathione S-transferase domain of AIMP2-DX2 through its substrate-binding domain. Furthermore, AIMP2-DX2 promoted the proliferation, migration, and invasion of nasopharyngeal carcinoma (NPC) cells by upregulating MMP-2 and MMP-994. In short, AIMP2-DX2 can not only serve as a potential biomarker for lung cancer, but also participate in tumorigenesis. Interestingly, the downregulation of AIMP2-DX2 expression by shRNA suppressed the epidermal growth factor receptor (EGFR)/ MAPK signaling pathway, thereby inhibiting glucose uptake and cancer cell growth ${ }^{95}$. Therefore, suppressing the expression of AIMP2-DX2 may be an effective strategy for treating cancer.

\section{AIMP3 and cancer}

As with AIMP2, AIMP3 was also a haploinsufficient tumor suppressor ${ }^{96}$. AIMP3 upregulated p53 by directly interacting with the FAT domains of ataxia telangiectasiamutated/ATM and Rad 3-related (ATM/ATR), thereby responding to DNA damage. Kim et al. observed several mutations of AIMP3 in chronic myeloid leukemia (CML) patients $^{97}$. Of these, the mutations at $\mathrm{Ser}^{87}, \mathrm{Val}^{98}$, and $\mathrm{Arg}^{144}$ blocked the interaction between AIMP3 and ATM, suggesting that these residues had important functions for p53 activation. Generally, AIMP3 and MRS interacted through the GST-homology domains in the $\mathrm{MSC}^{98}$. Under UV irradiation, MRS was phosphorylated at Ser662 by general control nonrepressed-2 (GCN2), causing a conformational change in MRS and the subsequent dissociation of AIMP3 from MRS. The dissociated AIMP3 translocated to the nucleus, where it participated in the DNA damage response. Hepatitis B virus X protein $(\mathrm{HBx})$ activated the lncRNA highly upregulated in liver cancer (HULC) promoter through cAMP-responsive elementbinding protein (CREB), thereby upregulating HULC expression in liver cancer HepG2 cells ${ }^{99}$. It was worth noting that the upregulated HULC promoted the proliferation of liver cancer cells by downregulating AIMP3. These results indicate that AIMP3 works as an important signaling molecule in tumorigenesis (Fig. 5).

AIMP3 expression was reduced in muscle-invasive bladder cancer (MIBC), resulting in impaired Tp53 transactivity and genomic instability ${ }^{100}$. Significantly, the reduction of AIMP3 increased the resistance of cancer cells to ionizing radiation. At the same time, AIMP3 expression predicted relapse and overall survival after radiotherapy, indicating that it could serve as a potential clinical biomarker for MIBC. Intriguingly, higher AIMP3 expression was associated with better survival in gastric and colon adenocarcinoma, but with poor survival in breast, lung, and liver cancers ${ }^{101}$. Therefore, more indepth research is needed to explore the relationship between AIMP3 and survival outcomes across cancer types.

\section{AIMPs and potential therapeutic interventions}

Meaningfully, several studies have focused on the potential clinical applications of AIMPs in cancer treatment. After 6 days of intravenous injection of recombinant AIMP1 in a mouse xenograft model bearing human stomach cancer cells, the tumor volume and weight decreased significantly ${ }^{102}$. The cells exhibiting an active cell cycle progression were reduced in tumor tissues of AIMP1-treated mice, and the blood levels of TNF- $\alpha$ and IL-1 $\beta$ were increased, indicating that AIMP1 might play an anti-tumor role by inducing tumor inhibiting cytokines. Furthermore, pharmacokinetic studies of a single intravenous injection in rats found that AIMP1 showed a low clearance and a low volume of distribution, and its half-life was $6 \mathrm{~min}$.

Interestingly, low-dose EMAP II induced Bcl-2/adenovirus E1B $19 \mathrm{kDa}$ protein-interacting protein 3 (BNIP3)mediated mitophagy by downregulating miR-24-3p, thereby enhancing the temozolomide cytotoxicity of $\mathrm{GSCs}^{103}$. Mice treated simultaneously with EMAP II, temozolomide and miR-24-3p inhibitor showed the smallest tumors and the longest survival rates, suggesting that the combined use of EMAP II and temozolomide might be a new approach for the treatment of glioma. Similarly, Awasthi et al. demonstrated that EMAP II enhanced the antitumor effects of sorafenib and 


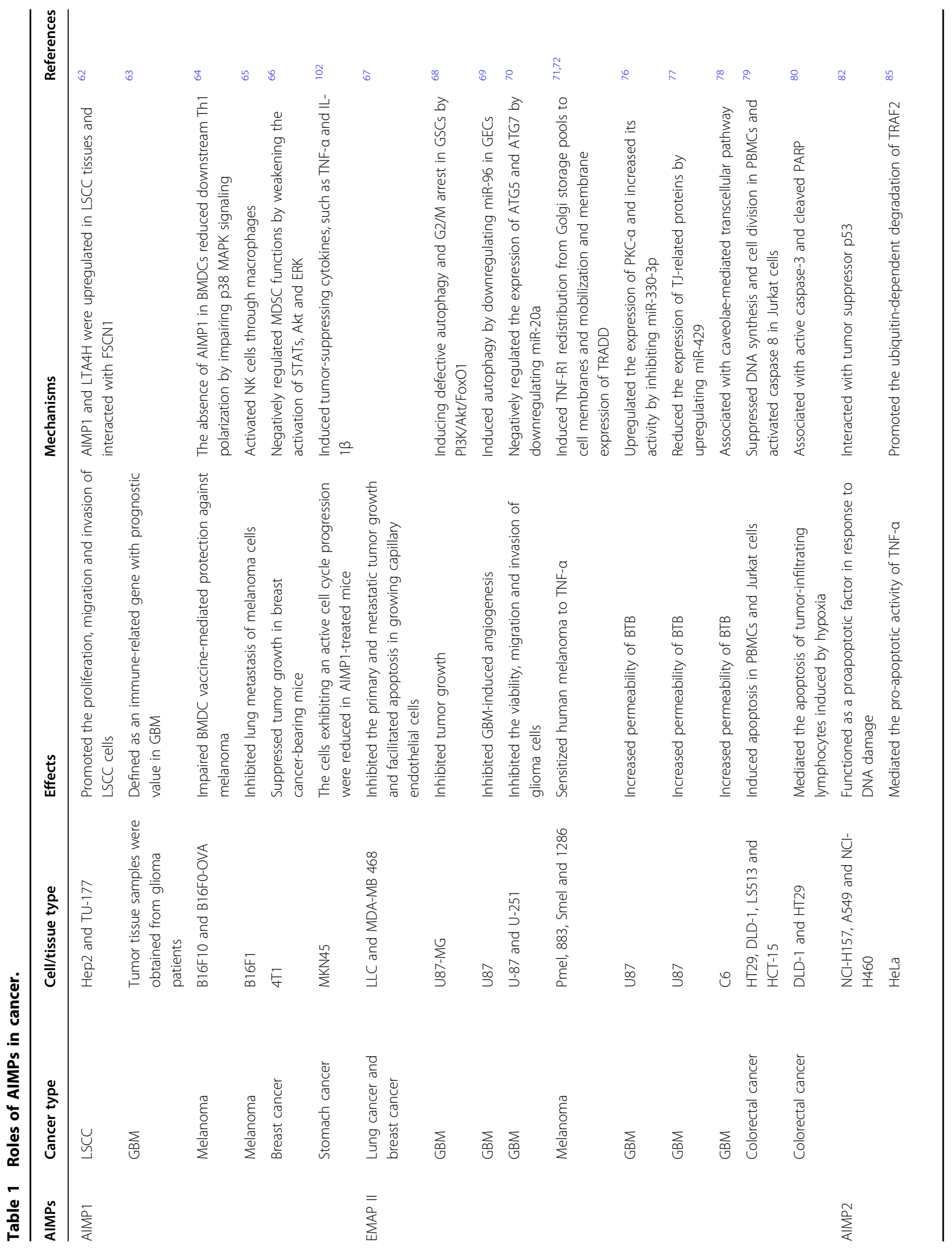




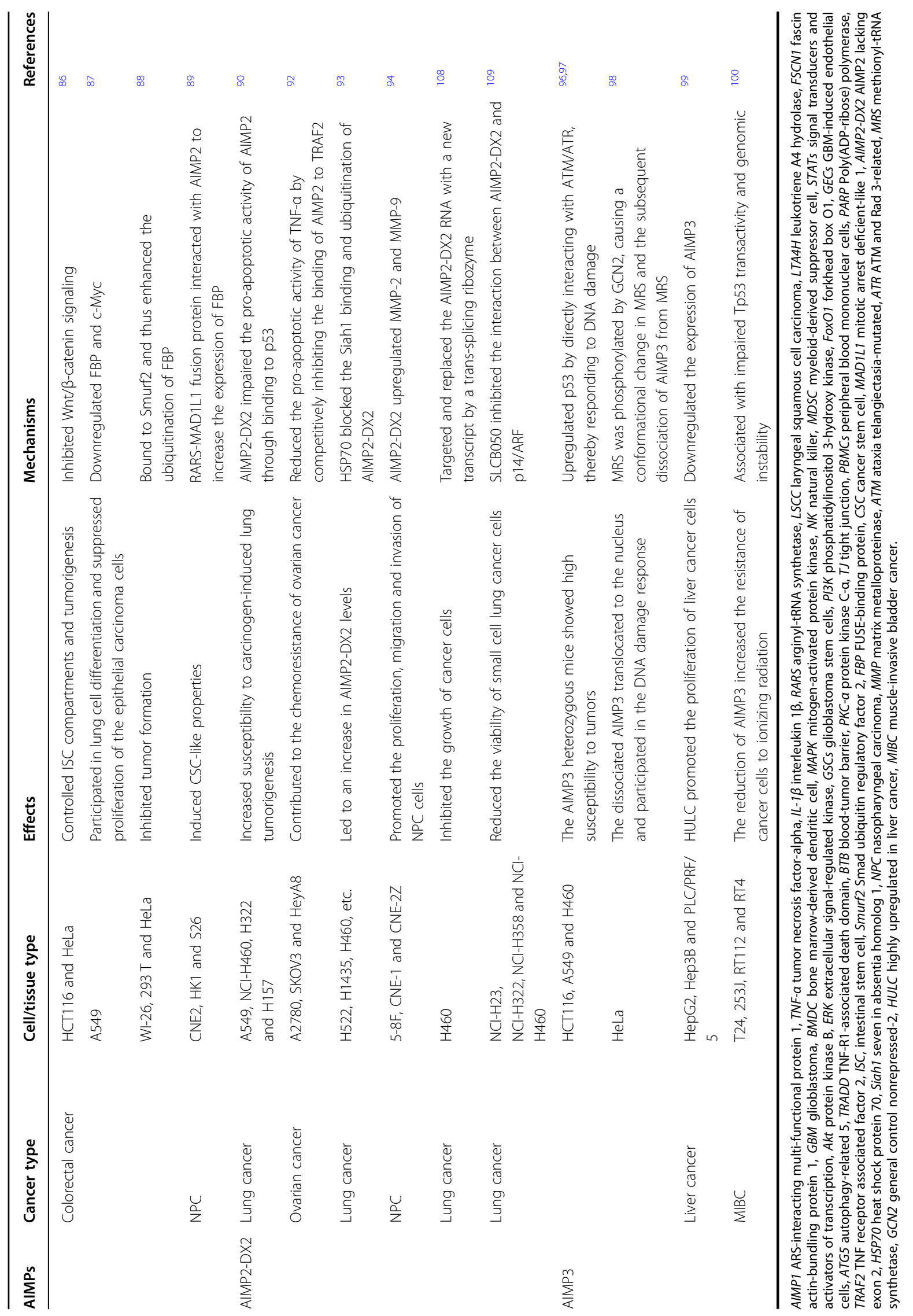


gemcitabine in pancreatic ductal adenocarcinoma $(\mathrm{PDAC})^{104}$. Compared to the control group, the combination therapy significantly improved animal survival. Notably, the serum EMAP II levels in patients with nonsmall cell lung cancer were significantly higher than in healthy subjects, and high serum EMAP II levels were associated with shorter survival, indicating that EMAP II could serve as a new biomarker for non-small cell lung cancer ${ }^{105}$.

In addition, many studies have attempted to suppress tumor development by targeting AIMP2-DX2 ${ }^{106,107}$. Won et al. effectively inhibited the growth of cancer cells by designing a trans-splicing ribozyme that targeted and replaced the AIMP2-DX2 RNA with a new transcript ${ }^{108}$. This ribozyme performed the RNA replacement by a high-fidelity trans-splicing reaction with the targeted residue of AIMP2-DX2 RNA, but did not work on normal AIMP2 transcript. AIMP2-DX2 eliminated oncogeneinduced cell death and aging by binding to and inhibiting $\mathrm{p} 14 / \mathrm{ARF}$, thereby promoting tumorigenesis ${ }^{109}$. Conspicuously, a novel compound, SLCB050, could reduce the viability of small cell lung cancer cells by inhibiting the interaction between AIMP2-DX2 and p14/ARF. Another inhibitor, BC-DXI-495, specifically bound to AIMP2-DX2 and blocked its interaction with HSP70, thus exerting an anti-tumor activity ${ }^{93}$. Furthermore, the label-free molecular probe based on G-quadruplex and strand displacement could sensitively and selectively detect AIMP2-DX2, which might be used for early diagnosis and monitoring the progression of relevant cancer ${ }^{110}$.

\section{Conclusion and future perspective}

Since the MSC plays an important role in protein synthesis, it is important to understand its structural features and the physiological functions of its components. Of these, AIMPs are generally considered as auxiliary proteins and play a scaffolding role during MSC assembly. In addition, AIMPs also participate in a spectrum of biological processes, including immune regulation, nervous system functions, angiogenesis, and genome stability, which are considered unexpected because AIMPs are classified as housekeeping proteins. Interestingly, most of the non-canonical functions are more or less related to tumorigenesis. In fact, AIMPs and their variants do play a vital role in tumor biology (Table 1). Specifically, AIMPs usually exert tumor-suppressive activities, while AIMP2-DX2 is involved in the development of cancer. Furthermore, certain AIMPs are also regarded as biomarkers for cancer prognosis. Therefore, the study of AIMPs in the context of cancer will be a promising field. However, more molecular mechanisms are needed to further clarify the relationship between AIMPs and cancer.
Indeed, as another component of the MSC, certain ARSs were also involved in tumorigenesis ${ }^{111,112}$. Genetic variants in several ARS genes have been reported to be associated with breast cancer risk in Chinese population $^{113}$. Recently, Zirin et al. found that ARSs were important mediators of Myc growth control in Drosophila, and their inhibitors killed human cells overexpressing Myc, suggesting that ARSs might serve as targets for treating Myc-driven cancers ${ }^{114}$. AIMPs are also emerging as closely related to cancer biology, and taking them into consideration will lead to a better understanding of tumorigenesis and contribute to the treatment of malignant tumors.

\section{Acknowledgements}

This research did not receive any specific grant from funding agencies in the public, commercial, or not-for-profit sectors.

\section{Author details}

'Department of Chinese Medicine, The First Affiliated Hospital of Zhengzhou University, Zhengzhou 450000, China. ${ }^{2}$ Department of Clinical Pharmacology, Xiangya Hospital, Central South University, Changsha 410000, China. ${ }^{3}$ Hunan Key Laboratory of Pharmacogenetics, Institute of Clinical Pharmacology, Central South University, Changsha 410000, China. ${ }^{4}$ Department of Pharmacy, The First Hospital of Changsha, Changsha 410005, China

Conflict of interest

The authors declare that they have no conflict of interest.

\section{Publisher's note}

Springer Nature remains neutral with regard to jurisdictional claims in published maps and institutional affiliations.

Received: 21 January 2020 Revised: 3 July 2020 Accepted: 6 July 2020 Published online: 24 July 2020

\section{References}

1. Kwon, N. H., Fox, P. L. \& Kim, S. Aminoacyl-tRNA synthetases as therapeutic targets. Nat. Rev. Drug Discov. 18, 629-650 (2019).

2. Rajendran, V., Kalita, P., Shukla, H., Kumar, A. \& Tripathi, T. Aminoacyl-tRNA synthetases: Structure, function, and drug discovery. Int. J. Biol. Macromol. 111, 400-414 (2018).

3. Guo, M., Ignatov, M., Musier-Forsyth, K., Schimmel, P. \& Yang, X. L. Crystal structure of tetrameric form of human lysyl-tRNA synthetase: Implications for multisynthetase complex formation. Proc. Natl Acad. Sci. USA 105, 2331-2336 (2008).

4. Fu, Y. et al. Structure of the ArgRS-GInRS-AIMP1 complex and its implications for mammalian translation. Proc. Natl Acad. Sci. USA 111, 15084-15089 (2014).

5. Cho, H. Y. et al. Symmetric assembly of a decameric subcomplex in human multi-tRNA synthetase complex via interactions between glutathione transferase-homology domains and aspartyl-tRNA synthetase. J. Mol. Biol. 431, 4475-4496 (2019).

6. Hei, Z., Wu, S., Liu, Z., Wang, J. \& Fang, P. Retractile lysyl-tRNA synthetaseAIMP2 assembly in the human multi-aminoacyl-tRNA synthetase complex. J. Biol. Chem. 294, 4775-4783 (2019).

7. Hahn, H. \& Park, S. H. The DRS-AIMP2-EPRS subcomplex acts as a pivot in the multi-tRNA synthetase complex. IUCrJ. 6, 958-967 (2019).

8. Cho, H. Y. et al. Assembly of multi-tRNA synthetase complex via heterotetrameric glutathione transferase-homology domains. J. Biol. Chem. 290, 29313-29328 (2015).

9. Schwarz, M. A., Lee, D. D. \& Bartlett, S. Aminoacyl tRNA synthetase complex interacting multifunctional protein 1 simultaneously binds Glutamyl-Prolyl- 
tRNA synthetase and scaffold protein aminoacyl tRNA synthetase complex interacting multifunctional protein 3 of the multi-tRNA synthetase complex. Int. J. Biochem. Cell Biol. 99, 197-202 (2018).

10. Mirzaei, M. et al. Upregulation of proteolytic pathways and altered protein biosynthesis underlie retinal pathology in a mouse model of Alzheimer's disease. Mol. Neurobiol. 56, 6017-6034 (2019).

11. Burgess, R. et al. The genetic landscape of epilepsy of infancy with migrating focal seizures. Ann. Neurol. 86, 821-831 (2019).

12. Kim, M. S., Kim, S. \& Myung, H. Degradation of AIMP1/p43 induced by hepatitis C virus E2 leads to upregulation of TGF-beta signaling and increase in surface expression of gp96. PLOS ONE 9, e96302 (2014).

13. Kim, H. et al. Estrogen receptor activation contributes to RNF146 expression and neuroprotection in Parkinson's disease models. Oncotarget $\mathbf{8}$ 106721-106739 (2017).

14. Ahn, J. et al. AlMP1 downregulation restores chondrogenic characteristics of dedifferentiated/degenerated chondrocytes by enhancing TGF-beta signal. Cell death Dis. 7, e2099 (2016).

15. Shukla, A. \& Das Bhowmik, A. Homozygosity for a nonsense variant in AIMP2 is associated with a progressive neurodevelopmental disorder with microcephaly, seizures, and spastic quadriparesis. J. Hum. Genet. 63, 19-25 (2018).

16. Kim, D. et al. AlMP3 deletion induces acute radiation syndrome-like phenotype in mice. Sci. Rep. 8, 15025 (2018).

17. Yin, K. et al. Using weighted gene co-expression network analysis to identify key modules and hub genes in tongue squamous cell carcinoma. Medicine 98, e17100 (2019).

18. Bronkhorst, I. H. et al. Effect of hypoxic stress on migration and characteristics of monocytes in uveal melanoma. JAMA Ophthalmol. 132, 614-621 (2014).

19. Atala, A. Re: Loss of expression of the tumour suppressor gene AIMP3 predicts survival following radiotherapy in muscle-invasive bladder cancer. J. Urol. 194, 1162-1163 (2015)

20. Kim, M. S. \& Kim, T. S. Aminoacyl tRNA synthetase-interacting multifunctional protein 1 acts as a novel B cell-activating factor in vitro and in vivo. J. Immunol. (Baltim., Md.: 1950) 194, 4729-4736 (2015).

21. Green, L. A. et al. HIV envelope protein gp120-induced apoptosis in lung microvascular endothelial cells by concerted upregulation of EMAP II and its receptor, CXCR3. Am. J. Physiol. Lung Cell. Mol. Physiol. 306, L372-L382 (2014).

22. Kim, H. \& Kang, S. J. Quantitative analysis of nasal transcripts reveals potential biomarkers for Parkinson's disease. Sci. Rep. 9, 11111 (2019).

23. Fan, X., Qi, B., Ma, L. \& Ma, F. Screening of underlying genetic biomarkers for ankylosing spondylitis. Mol. Med. Rep. 19, 5263-5274 (2019).

24. Ahn, S. S., Kim, J. O., Yoon, T., Song, J. J. \& Park, Y. B. Serum aminoacyl-tRNA synthetase-interacting multifunctional protein-1 can predict severe antineutrophil cytoplasmic antibody-associated vasculitis: a pilot monocentric study. Biomed. Res. Int. 2019, 7508240 (2019).

25. Burastero, S. E. \& Fabbri, M. Aminoacyl-tRNA synthetase-interacting multifunctional protein-1 (AIMP1): the member of a molecular hub with unexpected functions, including CD4 T cell homeostasis. Clin. Immunol. (Orlando, Fla.) 143, 207-209 (2012)

26. Clauss, M. et al. Lung endothelial monocyte-activating protein 2 is a mediator of cigarette smoke-induced emphysema in mice. J. Clin. Investig. 121, 2470-2479 (2011).

27. Ko, Y. G. et al. A cofactor of tRNA synthetase, $\mathrm{p43}$, is secreted to up-regulate proinflammatory genes. J. Biol. Chem. 276, 23028-23033 (2001).

28. Kwon, H. S. et al. Identification of CD23 as a functional receptor for the proinflammatory cytokine AIMP1/p43. J. cell Sci. 125, 4620-4629 (2012).

29. Kim, M. S., Lee, A., Cho, D. \& Kim, T. S. AlMP1 regulates TCR signaling and induces differentiation of regulatory $T$ cells by interfering with lipid raft association. Biochem. Biophys. Res. Commun. 514, 875-880 (2019).

30. Kao, J. et al. Endothelial monocyte-activating polypeptide II. A novel tumorderived polypeptide that activates host-response mechanisms. J. Biol. Chem 267, 20239-20247 (1992)

31. Quevillon, S., Agou, F., Robinson, J. C. \& Mirande, M. The p43 component of the mammalian multi-synthetase complex is likely to be the precursor of the endothelial monocyte-activating polypeptide II cytokine. J. Biol. Chem. 272 32573-32579 (1997).

32. Shalak, V. et al. The EMAPII cytokine is released from the mammalian multisynthetase complex after cleavage of its p43/proEMAPII component. J. Biol. Chem. 276, 23769-23776 (2001).

33. Lee, D. D. et al. Endothelial monocyte-activating polypeptide II mediates macrophage migration in the development of hyperoxia-induced lung disease of prematurity. Am. J. Respir. Cell Mol. Biol. 55, 602-612 (2016).
34. Lee, D. D., Hochstetler, A., Murphy, C., Lowe, C. W. \& Schwarz, M. A. A distinct transcriptional profile in response to endothelial monocyte activating polypeptide II is partially mediated by JAK-STAT3 in murine macrophages. Am. J. Physiol. Cell Physiol. 317, C449-c456 (2019).

35. Kotov, D. I., Mitchell, J. S. \& Pengo, T. TCR affinity biases th cell differentiation by regulating CD25, Eef1e1, and Gbp2. J Immunol. 202, 2535-2545 (2019).

36. Accogli, A. et al. Biallelic loss-of-function variants in AIMP1 cause a rare neurodegenerative disease. J. Child Neurol. 34, 74-80 (2019).

37. Accogli, A. et al. Pathogenic variants in AIMP1 cause pontocerebellar hypoplasia. Neurogenetics 20, 103-108 (2019).

38. labal, Z. et al. Missense variants in AIMP1 gene are implicated in autosomal recessive intellectual disability without neurodegeneration. Eur. J. Hum. Genet.: EJHG 24, 392-399 (2016)

39. BoAli, A. et al. Novel homozygous mutation of the AIMP1 Gene: a milder neuroimaging phenotype with preservation of the deep white matter. Pediatr. Neurol. 91, 57-61 (2019).

40. Zhu, X. et al. MSC p43 required for axonal development in motor neurons. Proc. Natl Acad. Sci. USA 106, 15944-15949 (2009).

41. Xu, H., Malinin, N. L., Awasthi, N., Schwarz, R. E. \& Schwarz, M. A. The N terminus of pro-endothelial monocyte-activating polypeptide II (EMAP \|) regulates its binding with the $C$ terminus, arginyl-tRNA synthetase, and neurofilament light protein. J. Biol. Chem. 290, 9753-9766 (2015).

42. Corti, O. et al. The p38 subunit of the aminoacyl-tRNA synthetase complex is a Parkin substrate: linking protein biosynthesis and neurodegeneration. Hum. Mol. Genet. 12, 1427-1437 (2003).

43. Lee, Y. et al. Parthanatos mediates AIMP2-activated age-dependent dopaminergic neuronal loss. Nat. Neurosci. 16, 1392-1400 (2013).

44. Yun, S. P. et al. VPS35 regulates parkin substrate AIMP2 toxicity by facilitating lysosomal clearance of AIMP2. Cell Death Dis. 8, e2741 (2017).

45. Jo, A., Lee, Y., Park, C. H. \& Shin, J. H. Deubiquitinase USP29 Governs MYBBP1A in the brains of Parkinson's disease patients. J. Clin. Med. https://doi.org/ 10.3390/jcm9010052 (2019)

46. Park, S. G. et al. Dose-dependent biphasic activity of tRNA synthetaseassociating factor, p43, in angiogenesis. J. Biol. Chem. 277, 45243-45248 (2002).

47. Wang, W. et al. p43 induces IP-10 expression through the JAK-STAT signaling pathway in HMEC-1 cells. Int. J. Mol. Med. 38, 1217-1224 (2016).

48. Berger, A. C. et al. Endothelial monocyte activating polypeptide II induces endothelial cell apoptosis and may inhibit tumor angiogenesis. Microvasc Res. 60, 70-80 (2000)

49. Schwarz, M. A., Zheng, H., Liu, J., Corbett, S. \& Schwarz, R. E. Endothelialmonocyte activating polypeptide II alters fibronectin based endothelial cell adhesion and matrix assembly via alpha5 beta1 integrin. Exp. Cell Res. 311 229-239 (2005).

50. Yuan, C. et al. Blockade of EMAP \| protects cardiac function after chronic myocardial infarction by inducing angiogenesis. J. Mol. Cell. Cardiol. 79, 224-231 (2015).

51. Gao, S. et al. Interaction of NS2 with AIMP2 facilitates the switch from ubiquitination to SUMOylation of M1 in influenza A virus-infected cells. J. Virol. 89, 300-311 (2015).

52. Liu, J. et al. Genetic variants in multisynthetase complex genes are associated with DNA damage levels in Chinese populations. Mutat. Res. 786, 8-13 (2016)

53. Kim, S. M., Jeon, Y., Kim, D. \& Jang, H. AlMP3 depletion causes genome instability and loss of stemness in mouse embryonic stem cells. Cell Death Dis. 9, 972 (2018).

54. Park, S. G. et al. Hormonal activity of AIMP1/p43 for glucose homeostasis. Proc. Natl Acad. Sci. USA 103, 14913-14918 (2006).

55. Ahn, J. et al. Aminoacyl-tRNA synthetase interacting multi-functional protein 1 attenuates liver fibrosis by inhibiting TGFbeta signaling. Int. J. Oncol. $\mathbf{4 8}$ 747-755 (2016).

56. Li, B. et al. MicroRNA-95 promotes myogenic differentiation by downregulation of aminoacyl-tRNA synthase complex-interacting multifunctional protein 2. Oncotarget 8, 111356-111368 (2017).

57. Kim, C., Park, J. M., Song, Y., Kim, S. \& Moon, J. HIF1alpha-mediated AIMP3 suppression delays stem cell aging via the induction of autophagy. Aging Cell 18, e12909 (2019).

58. Oh, Y. S. et al. Downregulation of lamin A by tumor suppressor AIMP3/p18 leads to a progeroid phenotype in mice. Aging Cell 9, 810-822 (2010).

59. Lee, Y. S. et al. Antitumor activity of the novel human cytokine AIMP1 in an in vivo tumor model. Molecules Cells 21, 213-217 (2006). 
60. Kim, S. S., Hur, S. Y., Kim, Y. R., Yoo, N. J. \& Lee, S. H. Expression of AIMP1, 2 and 3, the scaffolds for the multi-tRNA synthetase complex, is downregulated in gastric and colorectal cancer. Tumori 97, 380-385 (2011).

61. Bottoni, A. et al. Proteasomes and RARS modulate AIMP1/EMAP II secretion in human cancer cell lines. J. Cell. Physiol. 212, 293-297 (2007).

62. Gao, W. et al. Mass spectrometric analysis identifies AIMP1 and LTA4H as FSCN1-binding proteins in laryngeal squamous cell carcinoma. Proteomics 19, e1900059 (2019)

63. Cheng, W. et al. Bioinformatic profiling identifies an immune-related risk signature for glioblastoma. Neurology 86, 2226-2234 (2016).

64. Liang, D. et al. AIMp1 potentiates TH1 polarization and is critical for effective antitumor and antiviral immunity. Front. Immunol. 8, 1801 (2017).

65. Kim, M. S., Song, J. H., Cohen, E. P., Cho, D. \& Kim, T. S. Aminoacyl tRNA synthetase-interacting multifunctional protein 1 activates NK cells via macrophages in vitro and in vivo. J. Immunol. (Baltim., Md.: 1950) 198, 4140-4147 (2017)

66. Hong, $\mathrm{H}$. J. et al. Aminoacyl-tRNA synthetase-interacting multifunctional protein 1 suppresses tumor growth in breast cancer-bearing mice by negatively regulating myeloid-derived suppressor cell functions. Cancer Immunol. immunother. 65, 61-72 (2016).

67. Schwarz, M. A. et al. Endothelial-monocyte activating polypeptide II, a novel antitumor cytokine that suppresses primary and metastatic tumor growth and induces apoptosis in growing endothelial cells. J. Exp. Med. 190, 341-354 (1999).

68. Liu, J. et al. Anti-neoplastic activity of low-dose endothelial-monocyte activating polypeptide-II results from defective autophagy and G2/M arrest mediated by PI3K/Akt/FoxO1 axis in human glioblastoma stem cells. Biochemical Pharmacol. 89, 477-489 (2014).

69. Li, Z. et al. Endothelial-monocyte activating polypeptide II suppresses the in vitro glioblastoma-induced angiogenesis by inducing autophagy. Front. Mol. Neurosci. 10, 208 (2017).

70. Chen, J. et al. Low-dose endothelial-monocyte-activating polypeptide-I induced autophagy by down-regulating miR-20a in U-87 and U-251 glioma cells. Front. Cell. Neurosci. 10, 128 (2016).

71. Wu, P. C. et al. In vivo sensitivity of human melanoma to tumor necrosis factor (TNF)-alpha is determined by tumor production of the novel cytokine endothelial-monocyte activating polypeptide ॥ (EMAPII). Cancer Res. 59 205-212 (1999).

72. van Horssen, R., Rens, J. A., Schipper, D., Eggermont, A. M. \& ten Hagen, T. L. EMAP-II facilitates TNF-R1 apoptotic signalling in endothelial cells and induces TRADD mobilization. Apoptosis 11, 2137-2145 (2006).

73. Xie, H., Xue, Y. X., Liu, L. B. \& Liu, Y. H. Endothelial-monocyte-activating polypeptide II increases blood-tumor barrier permeability by downregulating the expression levels of tight junction associated proteins. Brain Res. 1319, 13-20 (2010).

74. Li, Z. et al. Roles of serine/threonine phosphatases in low-dose endothelial monocyte-activating polypeptide-Il-induced opening of blood-tumor barrier J. Mol. Neurosci. 57, 11-20 (2015).

75. Li, Z. et al. Low-dose endothelial monocyte-activating polypeptide-II increases permeability of blood-tumor barrier via a PKC-zeta/PP2A-dependent signaling mechanism. Exp. cell Res. 331, 257-266 (2015).

76. Liu, J. et al. The role of miR-330-3p/PKC-alpha signaling pathway in low-dose endothelial-monocyte activating polypeptide-II increasing the permeability of blood-tumor barrier. Front. Cell. Neurosci. 11, 358 (2017).

77. Chen, L. et al. MiR-429 regulated by endothelial monocyte activating polypeptide-II (EMAP-II) influences blood-tumor barrier permeability by inhibiting the expressions of ZO-1, occludin and claudin-5. Front. Mol. Neurosci. 11, 35 (2018)

78. Li, Z., Liu, Y. H., Xue, Y. X., Liu, L. B. \& Wang, P. Low-dose endothelial monocyte-activating polypeptide-ii increases permeability of blood-tumor barrier by caveolae-mediated transcellular pathway. J. Mol. Neurosci. 52 313-322 (2014).

79. Murray, J. C. et al. Colorectal cancer cells induce lymphocyte apoptosis by an endothelial monocyte-activating polypeptide-II-dependent mechanism. J. Immunol. (Baltim., Md.: 1950) 172, 274-281 (2004)

80. Youssef, M. M., Symonds, P., Ellis, I. O. \& Murray, J. C. EMAP-II-dependent lymphocyte killing is associated with hypoxia in colorectal cancer. $\mathrm{Br}$. J. Cancer 95, 735-743 (2006).

81. Choi, J. W., Um, J. Y., Kundu, J. K., Surh, Y. J. \& Kim, S. Multidirectional tumorsuppressive activity of AIMP2/p38 and the enhanced susceptibility of AIMP2 heterozygous mice to carcinogenesis. Carcinogenesis 30, 1638-1644 (2009).
82. Han, J. M. et al. AIMP2/p38, the scaffold for the multi-tRNA synthetase complex, responds to genotoxic stresses via p53. Proc. Natl Acad. Sci. USA 105, 11206-11211 (2008).

83. Cruceriu, D., Baldasici, O. \& Balacescu, O. The dual role of tumor necrosis factor-alpha (TNF-alpha) in breast cancer: molecular insights and therapeutic approaches. Cell Oncol (Dordr). 43, 1-18 (2020).

84. Lee, E. et al. The pleiotropic effects of TNFalpha in breast cancer subtypes is regulated by TNFAIP3/A20. Oncogene 38, 469-482 (2019).

85. Choi, J. W. et al. AIMP2 promotes TNFalpha-dependent apoptosis via ubiquitin-mediated degradation of TRAF2. J. Cell Sci. 122, 2710-2715 (2009).

86. Yum, M. K. et al. AIMP2 controls intestinal stem cell compartments and tumorigenesis by modulating wnt/beta-catenin signaling. Cancer Res. 76, 4559-4568 (2016).

87. Kim, M. J. et al. Downregulation of FUSE-binding protein and c-myc by tRNA synthetase cofactor p38 is required for lung cell differentiation. Nat. Genet. 34, 330-336 (2003)

88. Kim, D. G. et al. Oncogenic mutation of AlMP2/p38 inhibits its tumorsuppressive interaction with Smurf2. Cancer Res. 76, 3422-3436 (2016).

89. Zhong, Q. et al. The RARS-MAD1L1 fusion gene induces cancer stem cell-like properties and therapeutic resistance in nasopharyngeal carcinoma. Clin. Cancer Res. 24, 659-673 (2018).

90. Choi, J. W. et al. Cancer-associated splicing variant of tumor suppressor AIMP2/p38: pathological implication in tumorigenesis. PLoS Genet. 7 e1001351 (2011).

91. Jung, J. Y. et al. Ratio of autoantibodies of tumor suppressor AIMP2 and Its oncogenic variant is associated with clinical outcome in lung cancer. J. Cancer 8, 1347-1354 (2017)

92. Choi, J. W. et al. Splicing variant of AIMP2 as an effective target against chemoresistant ovarian cancer. J. Mol. cell Biol. 4, 164-173 (2012).

93. Lim, S. et al. Targeting the interaction of AIMP2-DX2 with HSP70 suppresses cancer development. Nat. Chem. Biol. 16, 31-41 (2020).

94. Cao, Q., Zhang, J. \& Zhang, T. AIMP2-DX2 promotes the proliferation, migration, and invasion of nasopharyngeal carcinoma cells. Biomed. Res. Int. 2018, 9253036 (2018).

95. Chang, S. H. et al. Lentiviral vector-mediated shRNA against AIMP2-DX2 suppresses lung cancer cell growth through blocking glucose uptake. Mol. Cells 33, 553-562 (2012).

96. Park, B. J. et al. The haploinsufficient tumor suppressor p18 upregulates p53 via interactions with ATM/ATR. Cell 120, 209-221 (2005).

97. Kim, K. J. et al. Determination of three-dimensional structure and residues of the novel tumor suppressor AIMP3/p18 required for the interaction with ATM. J. Biol. Chem. 283, 14032-14040 (2008).

98. Kwon, N. H. et al. Dual role of methionyl-tRNA synthetase in the regulation of translation and tumor suppressor activity of aminoacyl-tRNA synthetaseinteracting multifunctional protein-3. Proc. Natl Acad. Sci. USA 108, 19635-19640 (2011)

99. Du, Y. et al. Elevation of highly up-regulated in liver cancer (HULC) by hepatitis $B$ virus $X$ protein promotes hepatoma cell proliferation via downregulating p18. J. Biol. Chem. 287, 26302-26311 (2012).

100. Gurung, P. M. et al. Loss of expression of the tumour suppressor gene AIMP3 predicts survival following radiotherapy in muscle-invasive bladder cancer. Int. J. cancer 136, 709-720 (2015).

101. Hassan, M. K., Kumar, D., Naik, M. \& Dixit, M. The expression profile and prognostic significance of eukaryotic translation elongation factors in different cancers. PLOS ONE 13, e0191377 (2018).

102. Han, J. M., Myung, H. \& Kim, S. Antitumor activity and pharmacokinetic properties of ARS-interacting multi-functional protein 1 (AIMP1/p43). Cancer Lett. 287, 157-164 (2010).

103. Zhang, J. et al. Endothelial monocyte-activating polypeptide-II induces BNIP3mediated mitophagy to enhance temozolomide cytotoxicity of glioma stem cells via down-regulating MiR-24-3p. Front. Mol. Neurosci. 11, 92 (2018).

104. Awasthi, N., Zhang, C., Hinz, S., Schwarz, M. A. \& Schwarz, R. E. Enhancing sorafenib-mediated sensitization to gemcitabine in experimental pancreatic cancer through EMAP II. J. Exp. Clin. Cancer Res. 32, 12 (2013).

105. Sen, E., Ulger, F., Kaya, A., Akar, N. \& Gonullu, U. Serum endothelial monocyteactivating polypeptide-II: a novel biomarker in patients with non-small-cell lung cancer. Clin. lung cancer 9, 166-170 (2008).

106. Lee, H. S. et al. Chemical suppression of an oncogenic splicing variant of AIMP2 induces tumour regression. Biochemical J. 454, 411-416 (2013).

107. Hwang, S. K., Chang, S. H., Minai-Tehrani, A., Kim, Y. S. \& Cho, M. H. LentivirusAIMP2-DX2 shRNA suppresses cell proliferation by regulating Akt1 signaling 
pathway in the lungs of AIMP2 $(+) /(-)$ mice. J. Aerosol Med. Pulm. Drug Deliv. 26, 165-173 (2013).

108. Won, Y. S. \& Lee, S. W. Selective regression of cancer cells expressing a splicing variant of AIMP2 through targeted RNA replacement by transsplicing ribozyme. J. Biotechnol. 158, 44-49 (2012).

109. Oh, A. Y. et al. Inhibiting DX2-p14/ARF interaction exerts antitumor effects in lung cancer and delays tumor progression. Cancer Res. 76, 4791-4804 (2016).

110. Zang, R., Wang, X., Zhu, Y., Yao, T. \& Shi, S. Label-free molecular probe based on G-quadruplex and strand displacement for sensitive and selective detection and naked eye discrimination of exon 2 deletion of AIMP2. Chem Biol Drug Des. 93, 993-998 (2019).
111. Lee, J. M. \& Kim, T. Methionyl-tRNA synthetase is a useful diagnostic marker for lymph node metastasis in non-small cell lung cancer. Yonsei. Med. J. 60, 1005-1012 (2019).

112. Kim, S. B., Kim, H. R., Park, M. C., Cho, S. \& Goughnour, P. C. Caspase-8 controls the secretion of inflammatory lysyl-tRNA synthetase in exosomes from cancer cells. J. Cell Biol. 216, 2201-2216 (2017).

113. He, Y. et al. Potentially functional polymorphisms in aminoacyl-tRNA synthetases genes are associated with breast cancer risk in a Chinese population. Mol. Carcinog. 54, 577-583 (2015).

114. Zirin, J. et al. Interspecies analysis of MYC targets identifies tRNA synthetases as mediators of growth and survival in MYC-overexpressing cells. Proc Natl Acad Sci USA 116, 14614-14619 (2019). 